\title{
Validação da escala de Liderança para o Esporte: Versão Preferência dos Atletas
}

\author{
Validation of the leadership scale for sport: \\ Version of preference of athletes
}

\section{Validación de la escala de liderazgo para el deporte:}

Versión de preferencia de los atletas

José Augusto Evangelho

Hernandez

Universidade Estadual do

Rio de Janeiro

Rogério da Cunha Voser

Universidade Federal do

Rio Grande do Sul

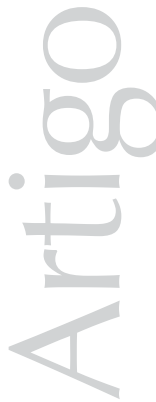


Resumo: Este estudo buscou promover uma validação de constructo da Escala de Liderança para o Esporte, de Chelladurai e Saleh $(1978,1980)$, versão preferência de liderança, com dados de 706 atletas brasileiros de futebol, judô, natação e atletismo de ambos os sexos. Os participantes da pesquisa pertenciam a diversos clubes esportivos do Rio Grande do Sul. Além disso, na tentativa de produzir uma validação de critério para o instrumento, foram testadas as relações entre a preferência de liderança dos atletas e a dependência e a variabilidade da tarefa, o gênero e a idade dos atletas, prescritas no Modelo Multidimensional de Liderança para o Esporte. As respostas dos atletas ao instrumento foram processadas através do SPSS com análise fatorial, coeficiente alfa de Cronbach e MANOVA. Em geral, os resultados forneceram razoável confirmação à estrutura original da escala, porém a fidedignidade da subescala comportamento autocrático ficou aquém do aceitável. Na discussão, são apresentadas sugestões para a reformulação de alguns itens.

Palavras-chave: Psicologia do esporte. Liderança. Avaliação psicológica. Validade do teste.

Abstract: This study sought to promote a construct validation of Chelladurai and Saleh's Leadership
Scale for Sports $(1978,1980)$, version preference of leadership, with data from 706 male and female
Brazilian athletes in soccer, judo, swimming and athletics. The survey participants were from various
sport clubs of Rio Grande do Sul; also in an attempt to produce a validation criterion for the instrument
relations between the preference for leadership of the athletes and the dependence and variability
of task, gender and age of the athletes were tested, as prescribed in the Multidimensional Model of
Leadership for Sport. The responses of the athletes to the instrument were processed through the
SPSS Factor Analysis, Cronbach alpha and MANOVA. In general, the results provided reasonable
confirmation to the original structure. However, the reliability of the autocratic behavior subscale
fell short of acceptable. In the discussion, suggestions are made for the reformulation of some items. Keywords: Sport psychology. Leadership. Psychological assessment. Test validity.

Resumen: Este estudio buscó promover una validación de constructo de la Escala de Liderazgo para el Deporte de Chelladurai y Saleh $(1978,1980)$, versión preferencia de liderazgo, con datos de 706 atletas brasileños de fútbol, judo, natación y atletismo de ambos los sexos. Los participantes de la pesquisa pertenecían a diversos clubes deportivos de Rio Grande do Sul. Además de eso, en la tentativa de producir una validación de criterio para el instrumento fueron testadas las relaciones entre la preferencia de liderazgo de los atletas y la dependencia y variabilidad de la tarea, el género y la edad de los atletas, prescritas en el Modelo Multidimensional de Liderazgo para el Deporte. Las respuestas de los atletas al instrumento fueron procesadas a través del SPSS con Análisis Factorial, Coeficiente Alfa de Cronbach y MANOVA. En general, los resultados proporcionaron razonable confirmación a la estructura original de la escala. Pero, el carácter fidedigno de la sub-escala Comportamiento Autocrático resultó mucho más que aceptable. En la discusión, son presentadas sugestiones para la reformulación de algunos ítems.

Palabras clave: Psicología del Deporte. Liderazgo. Evaluación Psicológica. Validez del Test.

A liderança é o processo comportamental que visa a influenciar sujeitos e grupos para que atinjam objetivos pretendidos. A liderança no esporte e no exercício engloba muitas dimensões, tais como a tomada de decisão, as técnicas motivacionais, o feedback, as relações interpessoais e a administração da equipe (Weinberg \& Gould, 2008).
Nos anos 20, os proponentes da teoria do traço psicológico sustentavam que líderes bemsucedidos possuiriam certas características de personalidade (tais como inteligência, otimismo, independência e autoconfiança) que garantiriam a liderança permanente, independentemente da situação. Essas ideias foram superadas pelo trabalho de 
Os comportamentos de liderança preferidos variariam conforme a personalidade, a idade, o sexo, a experiência e a modalidade desportiva dos membros do grupo, e os comportamentos reais do líder seriam indiretamente afetados pelas preferências do grupo e pelas situações (Chelladurai \& Saleh, 1980).
Stogdill (1948), que encontrou em líderes de sucesso grande diversidade de traços de personalidade.

Nas décadas seguintes, sob a égide do comportamentalismo, predominou a visão de que qualquer um poderia liderar, desde que aprendesse os comportamentos de líderes bem-sucedidos. Tharp e Gallimore (1976) observaram o treinador de basquetebol John Wooden, da Universidade da Califórnia, e registraram que usava poucos elogios e reprovações, e suas intervenções não excediam 20 segundos.

Mais recentemente, psicólogos influenciados por teorias contingenciais (Fiedler, 1964) têm adotado abordagens interacionais para estudar a liderança desportiva. Essas teorias consideram tanto os traços psicológicos quanto o ambiente na determinação da liderança (Chelladurai, 1978; Smoll \& Smith, 1989). O Modelo Multidimensional de Liderança no Esporte (MMLE) postulou que a efetividade do líder dependeria das características dos atletas e dos limites da situação. A satisfação e o desempenho dos atletas dependeriam de três tipos de comportamentos do líder: requeridos, preferidos e reais, sendo o comportamento requerido do líder, em muitas situações, determinado pelo próprio sistema organizacional. Os comportamentos de liderança preferidos variariam conforme a personalidade, a idade, o sexo, a experiência e a modalidade desportiva dos membros do grupo, e os comportamentos reais do líder seriam indiretamente afetados pelas preferências do grupo e pelas situações (Chelladurai \& Saleh, 1980). Para Chelladurai (1993), as características do líder, tais como personalidade, competência e experiência, afetariam diretamente esses comportamentos.
Chelladurai e Saleh desenvolveram a Escala de Liderança para o Esporte (ELE) para medir as preferências e as percepções dos atletas em relação ao comportamento de seus técnicos e à autopercepção dos técnicos. Na construção do instrumento, 160 estudantes de Educação Física responderam a 99 itens, que foram escolhidos e modificados do Leader Behavior Description Questionnaire (Halpin, 1957), do Supervisory Behavior Description Questionnaire (Fleishman, 1957a), do Leadership Opinion Questionnaire (Fleishman, 1957b) e do Leader Behavior Description Questionnaire-Form XII (Stogdill, 1963). As respostas foram submetidas à análise fatorial para componentes principais com rotação varimax. Uma solução para cinco fatores com 37 itens representou a melhor adequação aos dados assim denominados: treinamento, comportamento democrático, comportamento autocrático, apoio social e reforçamento do comportamento. Após, foram incluídos 7 itens para suprir comportamentos de instrução no fator treinamento e 6 itens relativos a apoio social, totalizando 50 itens.

No estudo de Chelladurai e Saleh (1980), participaram estudantes de Educação Física, de ambos os sexos, e atletas universitários masculinos de basquete, corrida, track and field e remo. Os estudantes expressaram suas preferências de liderança em esportes favoritos, e os atletas, suas preferências de liderança no esporte praticado e suas percepções sobre a liderança a que estavam submetidos. Através de análises fatoriais independentes para componentes principais com rotação varimax e os itens da ELE, os itens foram reduzidos para 40. Uma solução de cinco fatores explicou $41,2 \%$ da variância total dos dados; na preferência dos atletas, 39,3\%, e, na percepção dos atletas, 55,8\%. A validade de conteúdo baseada na interpretação da análise fatorial 
mostrou que essa solução foi mais estável e significativa. Os fatores foram assim descritos: treinamento e instrução, itens representativos de comportamentos que buscam melhorar o desempenho dos atletas dando instruções sobre habilidades, técnicas e estratégias; comportamento democrático, itens relativos à tomada de decisão, isto é, comportamentos que permitem aos atletas a participação nas decisões sobre metas do grupo, métodos de treino, táticas e estratégias de jogo; comportamento autocrático, comportamentos que enfatizam a autoridade pessoal e a tomada de decisão independente; apoio social, itens que representam consideração e respeito pessoal e demonstram preocupação com o bemestar e o relacionamento afetuoso com os atletas; feedback positivo, itens relacionados com o reconhecimento pelo desempenho no contexto esportivo. Originalmente, esse fator foi denominado reforçamento positivo.

Até então, os comportamentos do líder eram classificados em duas dimensões: consideração, que se refere aos comportamentos indicativos de amizade, confiança mútua, respeito e afeto no relacionamento entre o líder e os subordinados, e estrutura inicial, que se refere à organização e à clareza de papéis para os membros do grupo fornecidos com o treinamento e instrução (Fleishman, 1957a). A ELE passou a fornecer cinco dimensões do comportamento do líder esportivo. $\mathrm{O}$ treinamento e instrução e o apoio social corresponderiam a estrutura inicial e consideração, respectivamente; os comportamentos democráticos e autocráticos refletem o estilo de tomada de decisão adotado pelo técnico - o grau de participação permitido aos atletas. O apoio social e o feedback positivo seriam faces relacionadas a consideração, porém os mesmos são independentes. Apoio social é fornecido sem referência ao desempenho, enquanto feedback positivo é contingente ao mesmo (Chelladurai \& Saleh, 1978, 1980).

Os coeficientes alfa de Cronbach calculados por Chelladurai e Saleh (1980) para a preferência de liderança dos estudantes variaram de 0,79 a 0,66 entre as cinco subescalas da ELE; para a dos atletas, variaram de 0,83 a 0,45, e, para a percepção da liderança dos atletas, variaram de 0,93 a 0,79 . Além disso, foi realizado um testereteste com intervalo de 4 semanas com o grupo de estudantes, e a correlação entre as duas medidas variou de 0,82 a 0,71 entre as cinco subescalas.

Inúmeras pesquisas têm testado o MMLE. Algumas confirmaram que, quando o líder tem um estilo que se ajusta às preferências dos membros do grupo, a satisfação destes é elevada (Chelladurai, 1984; Weiss \& Friedrichs, 1986). Por outro lado, o estudo de Riemer e Toon (2001) não apoiou a hipótese de que a congruência entre a liderança preferida e a percebida seja preditora da satisfação dos atletas. Chelladurai e Carron (1983) encontraram relações entre as preferências de liderança e a maturidade atlética de atletas, e Sherman, Fuller e Speed (2000) detectaram diferenças vinculadas a gênero nas preferências de comportamentos de liderança em contextos esportivos. Também tem sido verificado que a liderança preferida pode ser mediada pelas características da tarefa desportiva praticada (Baker, Yardley, \& Côté, 2003; Chelladurai, 1990, 1993).

A dependência e a variabilidade são características da tarefa esportiva que podem estar relacionadas com a preferência de liderança dos atletas (Chelladurai \& Saleh, 1978). A tarefa é considerada interdependente quando o seu desempenho 
Chelladurai

(1978) propôs

que atletas

em esportes

interdependentes e abertos

prefeririam mais treinamento e instrução, e os atletas de modalidades independentes, efetivo requer interação com outras na equipe, quando o sucesso do conjunto depende da coordenação de todas as tarefas envolvidas. A variabilidade de tarefa refere-se ao grau de mudanças ambientais às quais o atleta precisa responder no desempenho. Tarefas de baixa variabilidade envolvem comportamentos motores fechados, relativamente estáveis, estáticos, em ambiente imutável, enquanto as de alta variabilidade envolvem comportamentos motores abertos, em que habilidades são usadas para responder a objetos que se movimentam e requerem ajustamento espaço-temporal contínuo do atleta (Gallahue, 2002).

Na teoria path-goal, House (1971) observou que o grau de ambiguidade, a interdependência e a variabilidade da tarefa poderiam determinar a adequação dos comportamentos de liderança. Esse autor sustentou que o comportamento da liderança poderia reduzir a ambiguidade e esclarecer os caminhos para as metas. Nesse caso, a liderança se relacionaria positivamente à satisfação e ao desempenho em tarefas mais ambíguas, mas seria desnecessária e redundante em tarefas não ambíguas. A estruturação e a supervisão resultariam em aumento da coordenação, da satisfação e do desempenho quando as tarefas fossem variadas e interdependentes. Chelladurai (1978) propôs que atletas em esportes interdependentes e abertos prefeririam mais treinamento e instrução, e os atletas de modalidades independentes, mais comportamento democrático e apoio social. O comportamento autocrático e o feedback positivo seriam mais preferidos pelos atletas de esportes interdependentes.

Não se tem conhecimento do desempenho psicométrico da ELE no Brasil, embora várias investigações tenham usado essa escala (De Lima, 2008; De Paiva, 2002; Franzen, 2000; Hernandez, Voser, \& Schardosin, 2001; Machado, Voser, \& Hernandez, 2004; Mancia Jr., 2008; Oliveira, Voser, \& Hernandez, 2004; Spier, 2008; Valvassori, Hernandez, \& Voser, 2008). Outros pesquisadores (Da Silva, Marques, \& Santos, 2008; Hoshino, Sonoo, \& Vieira, 2007; Lopes, Samulski, \& Noce, 2004; Simim, Paiva, \& Noce, 2005) utilizaram uma versão validada por Serpa, Lacoste, Antunes, Pataco e Santos (1989) em Portugal.

São raros os instrumentos estrangeiros que, utilizados na pesquisa em Psicologia do esporte no Brasil, possuem validação (Comissão de Esporte do CRP - SP, 2000). Além disso, até agora, nenhum instrumento de avaliação psicológica específico da área desportiva consta da lista homologada pelo Conselho Federal de Psicologia. Este estudo realizou uma validação de construto da Escala de Liderança para o Esporte (Chelladurai \& Saleh, 1978) com atletas brasileiros, na versão preferência de liderança, considerando o seu expressivo uso nas pesquisas brasileiras.

\section{Método}

\section{Participantes}

Foi usada uma amostra não probabilística de conveniência. Participaram deste estudo 706 atletas de ambos os sexos, das modalidades futebol, judô, natação e atletismo (Tabela 1) que pertenciam às equipes da Sociedade Ginástica de Porto Alegre, do Sport Club Ulbra, da Sociedade Caixeiros Viajantes, do Grêmio Náutico União, do Grêmio Foot-Ball Porto Alegrense, do Esporte Clube São José, do Grêmio Náutico Gaúcho, do Cerâmica 
Atlético Clube, do Tamoyo Futebol Clube, do Esperança Futebol Clube, do Estrela Futebol Clube e do Clube Esportivo Bento Gonçalves, cuja idade variou de 10 a 78 anos $(M=20$, $\mathrm{DP}=11,08)$.

Tabela 1. Distribuição da frequência dos participantes por sexo e modalidade esportiva

\begin{tabular}{|c|c|c|c|c|c|c|}
\hline & & \multicolumn{4}{|c|}{ Modalidade } & \multirow[t]{2}{*}{ Total } \\
\hline & & Futebol & Natação & Judô & Atletismo & \\
\hline \multirow[t]{2}{*}{ Sexo } & Masculino & 375 & 122 & 37 & 47 & 581 \\
\hline & Feminino & 0 & 60 & 43 & 22 & 125 \\
\hline Total & & 375 & 182 & 80 & 69 & 706 \\
\hline
\end{tabular}

Fonte: Dados da pesquisa

\section{Instrumento}

Foi testada a ELE de Chelladurai e Saleh (1980) na versão preferência de liderança. A escala foi traduzida mediante a técnica back translation. O instrumento contém 40 itens divididos em cinco subescalas: treinamento e instrução, apoio social, feedback positivo, comportamento democrático e comportamento autocrático. Para responder, foi usada uma Escala tipo Likert de cinco pontos: 1 (nunca), 2 (um pouco), 3 (às vezes), 4 (bastante) e 5 (sempre).

\section{Coleta de dados}

Os dados foram coletados nos locais de treinamento, às vezes, de forma individual, outras, coletiva. Os atletas foram informados dos objetivos da pesquisa, e leram e assinaram um Termo de Consentimento Livre e Informado. Para os menores de idade, a autorização foi fornecida pelos pais.

\section{Análise de dados}

Os dados foram analisados no SPSS, versão 17. A análise fatorial revelou a estrutura interna, o peso dos fatores e a complexidade estrutural dos itens da escala. Na solução fatorial rotada, usou-se o método varimax, visto que os fatores seriam ortogonais. Foi determinada a extração de cinco fatores, conforme o modelo de Chelladurai e Saleh (1980); também foi extraída uma solução inicial para componentes principais baseada em autovalores maiores do que 1. Além disso, a fidedignidade da ELE e de suas escalas foi avaliada pelo coeficiente alfa de Cronbach. Por fim, alguns testes que comparavam grupos e relacionavam variáveis foram produzidos, na tentativa de gerar evidências de validade de critério. A análise de variância comparou as respostas dos atletas masculinos de modalidades esportivas interdependentes (futebol) e independentes (judô, natação, atletismo). As mulheres não participaram desta análise, pois, nesta amostra, não havia mulheres praticantes de futebol. Para as tarefas independentes (judô, natação, atletismo), foi executada uma MANOVA com dois fatores (sexo dos atletas e variabilidade da tarefa). Quanto à 
variabilidade, o judô foi considerado tarefa aberta, e a natação e o atletismo, tarefas fechadas. $\mathrm{O}$ futebol, tarefa aberta, foi excluído desta análise, visto que era a única modalidade interdependente e constituída apenas de atletas do sexo masculino. Além disso, o coeficiente de correlação de Pearson investigou a relação entre a idade e a preferência de liderança dos atletas.

\section{Resultados}

O Teste Kaiser-Meyer-Olkin revelou uma medida de adequação amostral de 0,80, considerada meritosa. É desejável que o valor do índice KMO seja o mais próximo de 1. Isso quer dizer que o somatório dos coeficientes de correlação parcial entre as variáveis deve ser pequeno quando comparado ao somatório dos coeficientes de correlação observados (Kaiser \& Rice, 1974). O Teste de Esfericidade de Bartlett (1954) apresentou os seguintes resultados: $\chi^{2}(780)=4.971,54, \quad p=$ 0,000. Essa técnica serve para testar a hipótese nula de que a matriz de correlação é uma matriz identidade. Se essa hipótese não fosse rejeitada, o uso do modelo de análise fatorial deveria ser reavaliado.

A solução inicial da análise fatorial para componentes principais extraiu 11 fatores com autovalores superiores a 1, responsáveis por 51,5\% da variância. O Scree Test, de Cattell (1966), critério para determinar o número de fatores, revelou uma solução de cinco fatores possíveis, por isso, a quantidade de variância explicada a partir do quinto fator diminuiu consideravelmente (Figura 1).

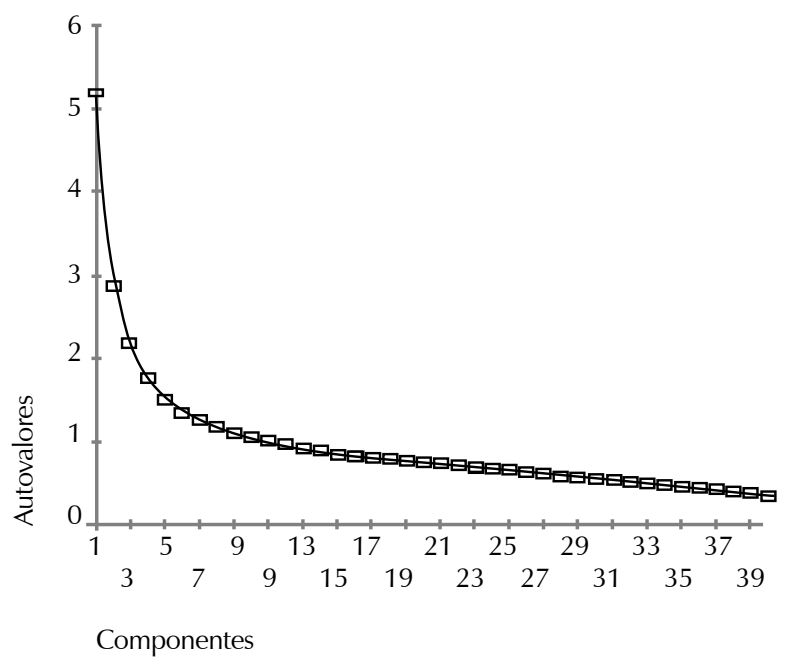

Figura 1. Componentes: Scree plot dos fatores da ELE extraídos pela análise de componentes principais

A solução para cinco fatores, conforme prescrito por Chelladurai e Saleh (1978), foi responsável por $33,8 \%$ da variância total. Com rotação varimax, a distribuição das cargas fatoriais dos itens pelos fatores foi a seguinte (Tabela 2): dos 13 itens previstos para a subescala treinamento e instrução, 11 carregaram mais forte no Fator 1; o item 14 carregou mais forte no Fator 2, e o item 23, no Fator 3; todos os 9 itens previstos para a subescala comportamento democrático carregaram mais 
forte no Fator 2; dos 8 itens previstos para a subescala apoio social, 5 carregaram mais forte no Fator 3; o item 22 ("Expresse sentimentos de afeto e de estima pelos atletas") carregou mais forte no Fator 5; o item 25 ("Estimule o atleta a falar abertamente e a confiar nele") carregou mais forte no Fator 1; o item 31 ("Tenha relações estreitas e informais com os atletas") carregou mais forte no Fator 4; todos os cinco itens previstos para a subescala comportamento autocrático carregaram mais forte no Fator 4, e todos os cinco itens previstos para a subescala feedback positivo carregaram mais forte no Fator 5. A consistência interna das respostas de preferência dos atletas foi examinada através do coeficiente alfa de Cronbach e variou de 0,72 a 0,56 para as subescalas da ELE, conforme a Tabela 2.

Tabela 2. Cargas fatoriais dos itens da ELE, solução de cinco fatores com rotação varimax

Componentes

ITENS

\begin{tabular}{llll}
\hline 1 & 2 & 3 & 4
\end{tabular}

1. Se preocupe que os atletas atinjam o máximo rendimento

2. Pergunte a opinião dos atletas sobre a tática que será usada em algumas provas

3. Ajude os atletas em seus problemas pessoais

4. Elogie um atleta, na frente do grupo, por ter desempenhado bem

5. Explique aos atletas a técnica e a tática da modalidade

6. Planeje os treinamentos sem levar em conta a opinião dos atletas

7. Ajude os atletas a solicionar os problemas que existem no grupo

8. Dê atenção especial para a correção dos erros dos atletas

9. Busque o apoio dos atletas antes de executar tarefaz importantes

10. Diga ao atleta se ele fez bem sua tarefa

11. Se assegure para que todos os atletas entendam o papel do treinador na equipe

12. Não tenha que dar explicações sobre suas decisões

13. Se preocupe com o bem-estar pessoal de seus atletas

14. Treine com cada atleta individualmente a técnica da modaliadade

15. Permita que os atletas opinem quando toma suas decisões

16. Esteja preocupado para que cada atleta seja reconhecido por desempenhar bem

17. Planeje antecipadamente o que vai fazer no treinamento

18. Estimule os atletas a fazer sugestões sobre como realizar o treino

19. Faça favores pessoais aos atletas

20. Explique a cada atleta o que deve ser feito e o que não deve ser feito no treino

21. Permita que os atletas definam seus próprios objetivos

22. Expresse sentimentos de afeto e de estima pelos atletas

23. Confie que cada atleta cumprirá suas obrigações nos mínimos detalhes

24. Permita que os atletas façam as coisas as suas maneiras ainda que cometam erros

25. Estimule o atleta a falar abertamente e a confiar nele

26. Mostre e treine os pontos fortes e os fracos de cada atleta

27. Se negue a mudar de opinião e imponha a sua

28. Demosntre seu reconhecimento por um atleta quando este joga bem

29. Dê instruções específicas a cada atleta sobre o que deve fazer em cada situação

30. Pergunte a opinião dos atletas sobre assuntos importantes do treino

31. Tenha relações estreitas e informais com os atletas

32. Se preocupe que o trabalho dos atletas seja coordenado

33. Permita que os atletas treinem como preferirem

34. Mantenha distância e não relacione muito com os atletas

35. Explique de que maneira cada atleta pode ajudar para que o grupo funcione bem

36. Se relacione como amigo com os atletas fora do campo

37. Reconheça o mérito de um atleta quando ele merece

38. Defina com detalhes o que espera de seus atletas nos treinos

39. Permita que os atletas decidam a tática que utilizarão em uma prova

40. Fale com tom automático sem permitir questionamentos

Autovalores

\%variância explicada

Alfa de Cronbach

Média

Desvio Padrão 
A análise de variância para um fator comparou os escores médios de preferência de liderança de atletas masculinos de tarefas interdependentes (futebol) e independentes (natação, atletismo e judô). Nas subescalas treinamento e instrução, feedback positivo e comportamento democrático, os atletas de esportes de tarefas independentes apresentaram escores médios mais elevados do que os interdependentes. Por outro lado, na subescala comportamento autocrático, os atletas de esportes de tarefas interdependentes apresentaram escores médios maiores do que os independentes; na subescala apoio social, porém, não houve diferença estatística significativa entre os tipos de tarefas (Tabela 3).

Tabela 3. Análise de variância para o fator Tipo de Tarefa e variável dependente subescalas da ELE

\begin{tabular}{|c|c|c|c|c|c|c|}
\hline Subescalas & Tipo de Tarefa & $\mathrm{n}$ & $M$ & DP & $\mathrm{F}$ & $\mathrm{P}$ \\
\hline \multirow[t]{2}{*}{ Treinamento e instrução } & Interdependente & 375 & 4,2 & 0,42 & 15.380 & 0,000 \\
\hline & Independente & 206 & 4,3 & 0,41 & & \\
\hline \multirow[t]{2}{*}{ Feedback positivo } & Interdependente & 375 & 4,0 & 0,57 & 8.574 & 0,004 \\
\hline & Independente & 206 & 4,2 & 0,61 & & \\
\hline \multirow[t]{2}{*}{ Apoio social } & Interdependente & 375 & 3,6 & 0,47 & 1.414 & 0,235 \\
\hline & Independente & 206 & 3,5 & 0,55 & & \\
\hline \multirow[t]{2}{*}{ Comportamento democrático } & Interdependente & 375 & 2,8 & 0,56 & 61.372 & 0,000 \\
\hline & Independente & 206 & 3,2 & 0,51 & & \\
\hline \multirow[t]{2}{*}{ Comportamento autocrático } & Interdependente & 375 & 2,3 & 0,74 & 10.019 & 0,002 \\
\hline & Independente & 206 & 2,1 & 0,65 & & \\
\hline
\end{tabular}

A MANOVA procurou verificar os efeitos das variáveis independentes sexo e variabilidade da tarefa sobre os escores médios de preferência de liderança dos atletas, exclusivamente das modalidades de tarefas independentes (judô, natação e atletismo). A interação do sexo dos atletas com a variabilidade da tarefa teve efeitos estatísticos significativos apenas sobre o comportamento democrático (Tabela 4), isto é, em atletas do sexo masculino com tarefas abertas, os escores médios em preferência do comportamento democrático foram maiores do que em atletas masculinos com tarefas fechadas e em femininos com tarefa aberta e fechada (Tabela 5). Não foram encontradas diferenças estatísticas significativas para o efeito principal do sexo dos atletas sobre os escores da ELE, porém foram encontradas para o efeito principal da variabilidade da tarefa. Os atletas praticantes de tarefas abertas mostraram escores médios de preferência de apoio social e comportamento democrático significativamente maiores do que os de tarefas fechadas;

Tabela 4. MANOVA para os fatores Sexo e Variabilidade da Tarefa e as variáveis dependentes subescalas da ELE

\begin{tabular}{llrr}
\hline Fonte & Variável Dependente & F & $P$ \\
\hline Sexo x variabilidade da tarefa & Treinamento e instrução & 1.663 & 0,198 \\
& Feedback positivo & 67 & 0,795 \\
& Apoio social & 52 & 0,820 \\
& Comportamento democrático & 4.314 & 0,039 \\
& Comportamento autocrático & 00 & 0,991
\end{tabular}


Tabela 5. Escores médios e Desvios-padrão de sexo dos atletas por variabilidade da tarefa em comportamento democrático

\begin{tabular}{|c|c|c|c|c|c|}
\hline & Sexo & Variabilidade da tarefa & $\mathrm{n}$ & M & DP \\
\hline \multirow{6}{*}{$\begin{array}{l}\text { Comportamento } \\
\text { democrático }\end{array}$} & Masculino & aberta & 37 & 3,4 & 0,48 \\
\hline & & fechada & 169 & 3,1 & 0,51 \\
\hline & & Total & 206 & 3,2 & 0,52 \\
\hline & Feminino & aberta & 43 & 3,2 & 0,50 \\
\hline & & fechada & 82 & 3,2 & 0,53 \\
\hline & & Total & 125 & 3,2 & 0,52 \\
\hline
\end{tabular}

Tabela 6. Análise de variância para variabilidade de tarefa nas subescalas da ELE

\begin{tabular}{|c|c|c|c|c|c|c|}
\hline & & $\mathrm{n}$ & M & DP & $\mathrm{F}$ & $\mathrm{p}$ \\
\hline \multirow{2}{*}{ Treinamento e instrução } & aberta & 80 & 4,3 & 0,37 & 149 & 0,699 \\
\hline & fechada & 251 & 4,3 & 0,43 & & \\
\hline \multirow[t]{2}{*}{ Feedback positivo } & aberta & 80 & 4,0 & 0,55 & 4.091 & 0,044 \\
\hline & fechada & 251 & 4,2 & 0,61 & & \\
\hline \multirow[t]{2}{*}{ Apoio social } & aberta & 80 & 3,6 & 0,47 & 4.737 & 0,030 \\
\hline & fechada & 251 & 3,5 & 0,56 & & \\
\hline \multirow[t]{2}{*}{ Comportamento democrático } & aberta & 80 & 3,3 & 0,50 & 5.635 & 0,018 \\
\hline & fechada & 251 & 3,1 & 0,51 & & \\
\hline \multirow[t]{2}{*}{ Comportamento autocrático } & aberta & 80 & 2,0 & 0,61 & 5.054 & 0,025 \\
\hline & fechada & 251 & 2,1 & 0,64 & & \\
\hline
\end{tabular}

por outro lado, os escores médios de preferência de feedback positivo e comportamento autocrático dos praticantes de tarefas abertas foram significativamente menores do que os de tarefas fechadas. Na preferência de treinamento e instrução, não houve diferença estatística significativa entre esses grupos (Tabela 6).

O coeficiente de correlação de Pearson revelou correlação positiva entre a idade dos atletas e os escores das subescalas treinamento e instrução $(r=0,14 ; p<0,01)$, feedback positivo ( $r$ $=0,12 ; p<0,01)$ e comportamento democrático $(r=0,17 ; p<0,01)$. Além disso, houve correlação negativa entre a idade dos atletas e os escores das subescalas apoio social $(r=$ - 0,08; $p<0,04)$ e comportamento autocrático $(r=-0,12 ; p<0,01)$. Em outros termos, conforme aumentou a idade dos atletas, aumentaram os escores nas subescalas treinamento e instrução, feedback positivo e comportamento democrático, e diminuíram nas subescalas apoio social e comportamento autocrático. 


\section{Discussão}

A primeira extração fatorial revelou 11 fatores com autovalores superiores a 1 , o que não corresponderia à estrutura preconizada por Chelladurai e Saleh (1980) para a ELE. A observação do Scree Test, porém, mostrou a possibilidade da adoção de cinco fatores como uma solução razoável, explicando 33,8\% da variância total, o que não diferiu muito do original (39,3\%).

Na análise dos itens, alguns não representaram os fatores conforme a prescrição. Os itens 14 e 23, que pertenceriam ao fator treinamento e instrução, carregaram mais forte, respectivamente, nos fatores comportamento democrático e apoio social. Comparando esses itens com suas formulações originais, notaram-se algumas imprecisões de tradução que podem ter alterado o seu sentido e a compreensão dos respondentes. A frase "Treine com cada atleta individualmente a técnica da modalidade" (item 14) poderia levar o leitor a pensar em uma concessão do treinador e, portanto, em uma conduta democrática. Além disso, as expressões "com cada" e "individualmente" são redundantes. Sugere-se que esse item seja reformulado para "Explique a cada atleta as técnicas e táticas do esporte", que representaria melhor a instrução. Também é possível que "Confie que cada atleta cumprirá suas obrigações nos mínimos detalhes" (item 23) se associe à ideia de confiança do treinador no indivíduo e, em consequência, a apoio social. Da mesma forma, sugere-se a troca para "Espere que cada atleta execute a sua tarefa nos mínimos detalhes", que evidenciaria a expectativa e a exigência do treinador em relação à tarefa.

Na subescala apoio social, três itens não apresentaram o desempenho esperado. $\mathrm{O}$ item 22 ("Expresse sentimentos de afeto e de estima pelos atletas") carregou mais forte no feedback positivo. A diferença entre os fatores apoio social e feedback positivo está baseada no fato de que este último se refere a comportamentos de reconhecimento e consideração quanto ao desempenho do atleta na tarefa. Por outro lado, apoio social se refere ao respeito à pessoa, independentemente da tarefa. Embora a formulação atual pareça adequada ao conceito de apoio social, os resultados não a confirmaram. Sugere-se uma reformulação do item para "Expresse o afeto que sente pelos seus atletas", que se parece mais com o item original e poderia ser testada nas próximas investigações. O item 25 ("Estimule o atleta a falar abertamente e a confiar nele") carregou mais forte no treinamento e instrução, e sugere-se sua alteração para "Estimule o atleta a confiar nele". Da mesma forma, recomenda-se que o item 31 ("Tenha relações estreitas e informais com os atletas"), que carregou mais forte no comportamento autocrático, seja reformulado para "Proponha relações próximas e informais com os atletas".

Para Dwyer e Fischer (1988), as consistências internas foram completamente aceitáveis para as subescalas treinamento e instrução, comportamento democrático e feedback positivo. No entanto, para esses autores, a consistência interna da subescala apoio social foi classificada como marginal e, para a subescala comportamento autocrático, foi muito baixa e inaceitável. No estudo de Chelladurai e Saleh (1980), os coeficientes foram declarados aceitáveis, mas o comportamento autocrático preferido pelos atletas teve índice muito baixo $(0,45)$. Salminen e Liukkonen (1994) também encontraram baixa consistência interna na subescala comportamento autocrático, e, nesse caso, a versão usada foi a do treinador. Em geral, com os dados atuais, os alfas de Cronbach obtidos foram similares aos encontrados antes, não foram 
os mais satisfatórios, entretanto, segundo Chelladurai e Saleh (1980), esses índices seriam melhores na versão percepção de liderança.

Na comparação entre os tipos de tarefas, os atletas de futebol (interdependentes) preferiram mais o comportamento autocrático do que os atletas de natação, atletismo e judô (independentes). Isso estaria de acordo com os achados de Chelladurai (1978), de Chelladurai e Saleh (1978) e de Terry e Howe (1984). Essas diferenças de preferência de liderança podem estar baseadas nas exigências organizativas e estruturais das modalidades coletivas e individuais que requerem estilos de liderança de natureza autocrática e democrática, respectivamente, segundo Alves (2000).

Os atletas independentes superaram os interdependentes na preferência de treinamento e instrução, feedback positivo e comportamento democrático. House (1971), na sua teoria path-goal de liderança, postulou que, quando as tarefas são abertas (de alta variabilidade) e independentes, os praticantes preferirão melhor estrutura e supervisão mais próxima ou um estilo de liderança de treinamento e instrução. Nesta pesquisa, apenas o judô foi caracterizado como tarefa independente e aberta. Apoiando a teoria de House, a média de preferência de treinamento e instrução dos judocas foi a que mais se diferenciou da média do futebol, modalidade interdependente e aberta.

Divergindo dos achados atuais e da teoria path-goal, Chelladurai (1978) sustentou que atletas de esportes interdependentes e abertos preferem mais treinamento e instrução. Além disso, atletas de esportes independentes preferem mais apoio social. Concordando, Terry (1984) reportou que atletas interdependentes preferiram mais treinamento e instrução, comportamento autocrático e feedback positivo e menos comportamento democrático e de apoio social. Reforçando esses resultados, Martens (2004) afirmou que os atletas de esportes interdependentes preferem líderes mais orientados para a tarefa (treinamento e instrução).

No presente estudo, através da MANOVA, não foram encontradas diferenças estatísticas significativas do efeito principal do sexo dos atletas sobre a sua preferência de liderança, semelhante a estudos anteriores (Bolkiah \& Terry, 2001; Chelladurai, Haggerty, \& Baxter, 1989; Terry \& Howe, 1984). Williams (1980), examinando as características de personalidade de mulheres atletas, constatou que eram mais agressivas, independentes, emocionalmente mais estáveis e mais orientadas para a tarefa do que as não atletas. As atletas do sexo feminino, portanto, apresentariam atributos psicológicos muito semelhantes às mentes masculinas, o que explicaria, em parte, o resultado encontrado neste estudo. Contudo, esse tem sido um aspecto controverso nessa linha de pesquisa, haja vista que Terry (1984) descobriu que homens preferiram mais comportamento autocrático. Da mesma forma, outros pesquisadores descobriram que os atletas do sexo feminino têm preferência por estilos de liderança mais participativos (comportamento democrático), enquanto os do sexo masculino preferem o comportamento autocrático, o treinamento e instrução e o feedback positivo (Chang, 2006; Chelladurai \& Arnott, 1985; Chelladurai \& Saleh, 1978; Weinberg \& Gould, 2008).

Também foram encontradas diferenças estatísticas significativas do efeito principal da variabilidade da tarefa (aberta ou fechada) sobre a preferência de liderança. Os atletas de tarefas abertas preferiram 
mais apoio social e comportamento democrático, e os de tarefas fechadas, mais feedback positivo e comportamento autocrático. Por outro lado, não houve diferença entre tarefas abertas e fechadas na preferência de treinamento e instrução. No entanto, em Chelladurai e Saleh (1978), atletas em tarefas abertas preferiram mais treinamento e instrução e feedback positivo do que em esportes fechados. Entretanto, outros estudos não encontraram efeito da variabilidade da tarefa sobre a preferência de liderança (Bolkiah \& Terry, 2001; Terry \& Howe, 1984).

Esta pesquisa, através da MANOVA, identificou um efeito significativo da interação das variáveis sexo e variabilidade de tarefa sobre a preferência de liderança na dimensão comportamento democrático. Beam, Serwatka e Wilson (2004) encontraram que, em tarefas fechadas, atletas do sexo feminino preferiram mais comportamento democrático do que atletas masculinos. Esses resultados confirmaram estudos anteriores de Chelladurai e Arnott (1985), Chelladurai e Saleh (1978) e Terry (1984), nos quais as atletas mulheres preferiram mais o líder democrático, e os atletas homens, o autocrático. No entanto, em tarefas abertas, atletas do sexo masculino preferiram mais comportamento democrático do que atletas femininos e também mais do que atletas masculinos em tarefas fechadas. Isso coincidiu com os resultados atuais, em que atletas homens em tarefas abertas preferiram mais comportamento democrático do que homens em tarefas fechadas e mulheres (em ambas as tarefas).

Uma contribuição importante para a compreensão das controvérsias em preferência de liderança esportiva foi dada por Riemer e Chelladurai (1995). Esses pesquisadores ponderaram que, embora as pesquisas realizadas incluíssem vários esportes que diferiam em dependência e variabilidade da tarefa, também estes poderiam divergir em outros aspectos situacionais, tais como: tamanho do grupo, popularidade do esporte e grau de pressão para o desempenho. Dessa forma, os resultados relativos ao tipo de tarefa poderiam ser confundidos pelos efeitos de outras variáveis situacionais sobre a preferência de liderança. Como essas variáveis não foram controladas, é possível que esse tipo de interferência tenha afetado os resultados da presente pesquisa.

$\mathrm{Na}$ investigação atual, a idade dos atletas variou de 10 a 78 anos e apresentou uma correlação positiva com a preferência em treinamento e instrução e feedback positivo. Para o treinamento e instrução, os resultados de Chelladurai e Carron (1983) mostraram que a preferência dos atletas nas escolas secundárias diminuiu de níveis de idades inferiores (13 anos) para níveis superiores (18 anos) e, após, aumentou para os universitários. O crescimento da preferência nesse ultimo estágio foi consistente com os relatos de Chelladurai (1978), nos quais atletas universitários, os mais experientes, preferem mais o treinamento e instrução do que os menos experientes. Essas preferências poderiam refletir o fato de que os mais velhos se tornariam mais compromissados com seus desempenhos e, portanto, mais orientados para suas metas.

Contrariando os resultados relatados na literatura, no estudo atual, a idade dos atletas associou-se positivamente a comportamento democrático e negativamente a comportamento autocrático da liderança. No entanto, tem sido observado que, conforme cresce a experiência no esporte, os atletas mostram um aumento na preferência pelo comportamento autocrático da liderança (Chelladurai \& Carron, 1982, 1983; Chelladurai \& 
Saleh, 1978). Chelladurai e Carron (1983) sugeriram que o relacionamento entre idade e preferência por comportamento autocrático pode ser devido ao fato de os atletas se socializarem em um sistema (âmbito esportivo) que, em geral, é autocrático, e, em consequência, preferirem menos participação. Confirmando essas tendências, no estudo de Serpa (1990), os atletas mais jovens apresentaram preferência por comportamento democrático e rejeição ao comportamento autocrático. Além disso, contrário aos resultados deste estudo, a preferência pelo apoio social aumenta com a experiência no esporte, segundo Chelladurai e Carron (1982, 1983). Por outro lado, nas pesquisas de Terry (1984) e de Terry e Howe (1984), nenhuma diferença significativa na preferência da liderança relacionada com a idade dos atletas foi encontrada.

\section{Conclusão}

Conforme a análise da estrutura da ELE, os itens representaram bem os fatores, haja vista que, em três destes (feedback positivo, comportamento democrático e comportamento autocrático), a estrutura original se confirmou integralmente. É verdade que problemas foram detectados em cinco itens dos fatores treinamento e instrução e apoio social. Nas próximas utilizações do instrumento, as sugestões de reformulação desses itens talvez possam solucionar, em parte, essas fragilidades. Historicamente, a subescala apoio social tem apresentado desempenho psicométrico fraco. Em geral, a ELE na versão preferência de liderança tem mostrado desempenho mais fraco do que na percepção de liderança. Sugere-se, para futuras investidas, a verificação dessa última versão da escala.

Na composição da amostra, a ausência de praticantes de esportes interdependentes com tarefas fechadas de ambos os sexos e de atletas femininas de esportes interdependentes com tarefas abertas não possibilitou uma comparação entre todas as condições possíveis. Contudo, algumas evidências de validade de critério para a escala foram geradas. O instrumento mostrou competência para detectar diferenças de preferência de liderança entre atletas de esportes interdependentes e independentes quanto a aspectos da tomada de decisão (comportamentos autocráticos e democráticos). Além disso, a relação da idade dos atletas às suas preferências de liderança apoiou parcialmente o modelo na dimensão treinamento e instrução.

Como a validação de um instrumento de medição psicológica é um processo alimentado pela acumulação gradativa de conhecimentos, acredita-se que esta experiência tenha sido uma pequena e estimulante contribuição para a continuidade do mesmo. No entanto, será imprescindível a realização de novos estudos confirmatórios que testem e promovam os ajustes necessários a ELE no contexto do esporte brasileiro. 


\section{José Augusto Evangelho Hernandez}

Doutor em Psicologia pela Universidade Federal do Rio Grande do Sul, Especialista em Psicologia do Esporte, docente do Departamento de Fundamentos da Psicologia na Universidade do Estado do Rio de Janeiro, Rio de Janeiro - RJ - Brasil. E-mail: hernandz@portoweb.com.br

\section{Rogério da Cunha Voser}

Doutor em Ciências da Saúde pela Pontificia Universidade Católica do Rio Grande do Sul, docente da Escola Superior de Educação Física na Universidade Federal do Rio Grande do Sul - RS - Brasil.

E-mail: rogerio.voser@ufrgs.br

\section{Endereço para envio de correspondência:}

Rua São Francisco Xavier, 524 - Instituto de Psicologia, 10o andar, sala 10006, bloco B Maracanã - Rio de Janeiro -RJ CEP: 20550-900

Recebido 22/12/2010, Aprovado 15/10/2011.

\section{Referências}

Alves, J. (2000). Liderazgo y clima organizacional. Revista de Psicología del Deporte, IX(1-2), 122-133.

Baker, J., Yardley, J., \& Côté, J. (2003). Coach behaviors and athlete satisfaction in team and individual sports. International Journal of Sport Psychology, 34, 226-239.

Bartlett, M. S. (1954). A note on the multiplying factors for various chi square approximations. Journal of the Royal Statistics Society, 16(Series B), 296-298.

Beam, J. W., Serwatka, T. S., \& Wilson, W. J. (2004). Preferred leadership of NCAA division I and II intercollegiate studentathletes. Journal of Sport Behavior, 27(1), 3-17.

Bolkiah, S., \& Terry, P. C. (2001). Coaching preferences of athletes in Brunei Darussalam and Great Britain: A cross-cultural test of path-goal theory. In International Society of Sport Psychology, 10th World Congress of Sport Psychology, Skiathos, Greece. Recuperado em 04 agosto, 2009, de http://eprints.usq.edu. au/4406/1/Bolkiah_Terry.pdf.

Cattell, R. B. (1966). The scree test for the number of factors. Multivariate Behavioral Research, 1(2), 245-276.

Chang, D-C. (2006). Effects of gender and age on preferred leadership behaviors of intercollegiate basketball players in Taiwan. Recuperado em 20 julho, 2009 de http://192.192.169.230/edu_paper/data_image/ g0000487/0n14/20061200/p0000284.pdf.

Chelladurai, P. (1978). A contingency model of leadership in athletics. Doctoral dissertation. University of Waterloo, Canada.
Chelladurai, P. (1984). Discrepancy between preferences and perceptions of leadership behavior and satisfaction of athletes in varying sports. Journal of Sport Psychology, 6, 27-41.

Chelladurai, P. (1990). Leadership in sports: A review. International Journal of Sport Psychology, 21, 328-354.

Chelladurai, P. (1993). Leadership. In R. Singer, M. Murphey \& L. Tennant (Eds.), Handbook of research on sport psychology (pp.647-671). New York: McMillan Publishing Company.

Chelladurai, P., \& Arnott, M. (1985). Decision styles in coaching: Preferences of basketball players. Research Quarterly for Exercise and Sport, 56(1), 15-24.

Chelladurai, P., \& Carron, A. V. (1983). Athletic maturity and preferred leadership. Journal of Sport Psychology, 5, 371-380.

Chelladurai, P., Haggerty, T. R., \& Baxter, P. R. (1989). Decision style choices of university basketball coaches and players. Journal of Sport \& Exercise Psychology, 11, 201-215.

Chelladurai, P., \& Saleh, S. D. (1978). Preferred leadership in sports. Canadian Journal of Applied Sports Sciences, 3, 85-92.

Chelladurai, P., \& Saleh, S. D. (1980). Dimensions of leader behavior in sports: Development of leadership scale. Journal of Sport Psychology, 2, 34-45.

Comissão de Esporte do CRP - SP. (2000). A avaliação psicológica no esporte ou os perigos da normatização e da normalização. In K. Rúbio (Org.), Psicologia do esporte: interfaces, pesquisa e intervenção. São Paulo: Casa do Psicólogo. 


\section{Referências}

Da Silva, N. F., Marques, M. G., \& dos Santos, G. A. (2008). Estilo de liderança de uma equipe de futebol juvenil do Vale do Paranhana. Revista Digital, 123. Recuperado em 28 maio, 2009, dehttp://www.efdeportes.com/indic123.htm.

De Lima, T. R. (2008). Liderança no judô: autopercepção dos treinadores e preferência dos atletas. Trabalho de conclusão de curso, Educação Física, Faculdades Integradas São Judas Tadeu, Porto Alegre, RS

De Paiva, L. F. F. (2002). A preferência dos jogadores de futsal com relação ao estilo de treinador. Monografia de especialização, Curso de Especialização em Esporte com Ênfase em Futsal, Educação Física, ULBRA, Canoas, RS.

Dwyer, J. J. M., \& Fischer, D. G. (1988). Psychometric propertie of the coach's version of leadership scale for sports. Perceptual and Motor Skills, 67, 795-798.

Fiedler, F. E. (1964). A contingency model of leadership effectiveness. Advances in experimental social psychology, 1, 149-190.

Fleishman, E. A. (1957a). A leader behavior description for industry. In R. M. Stogdill \& A. E. Coons (Eds.), Leader behavior: Its description and measurement. Columbus: The Ohio State University, Bureau of Business Research.

Fleishman, E. A. (1957b). The leadership opinion questionnaire In R. M. Stogdill \& A. E. Coons (Eds.), Leader behavior: Its description and measurement. Columbus: The Ohio State University, Bureau of Business Research.

Franzen, C. E. (2000). A preferência de liderança em atletas de esportes coletivos e individuais. Trabalho de conclusão de curso, Curso de Psicologia, ULBRA, Canoas, RS.

Gallahue, D. L. (2002). A classificação das habilidades de movimento: um caso para modelos multidimensionais. Revista da Educação Física, 13(2), pp. 105-111.

Halpin, A. W. (1957). The leader behavior and effectiveness of aircraft commanders. In R. M. Stogdill \& A. E. Coons (Eds.). Leader behavior: Its description and measurement. Columbus: The Ohio State University, Bureau of Business Research.

Hernandez, J. A. E., Voser, R. da C., \& Schardosin, A. L. T. (2001). A expectativa da liderança em atletas de modalidades esportivas coletivas e individuais: questões de gênero. In 1 Pré Conbrace. Anais. Pelotas, RS: Editora e Gráfica Universitária - UFPel.

Hoshino, E. F.; Sonoo, C. N., \& Vieira, L. F. (2007). Perfil de liderança: uma análise no contexto esportivo de treinamento e competição. Revista da Educação Física, 18(1), 77-83.

House, R. J. (1971). A path-goal theory of leader effectiveness. Administrative Science Quarterly, 16, 321-339.

Kaiser, H. F., \& Rice, J. (1974). Little Jiffy, Mark IV. Educational and psychological Measurement, 34(1), 111-117.

Lopes, M., Samulski, D., \& Noce, F. (2004). Análise do perfi ideal do treinador de voleibol das seleções brasileiras juvenis. Revista Brasileira de Ciências e Movimento, 12(4), 51-55.

Machado, S. do P., Voser, R. da C., \& Hernandez, J. A. E. (2004). A preferência dos jogadores de futsal com relação ao estilo de liderança do treinador. In Livro de Resumos, XV Salão de Iniciação Científica e XII Feira de Iniciação Científica. Porto Alegre, RS: UFRGS

Mancia Jr., M. G. G. (2008). O estilo de liderança preferido dos nadadores. Trabalho de conclusão de curso, Educação Física, Faculdades Integradas São Judas Tadeu, Porto Alegre, RS

Martens, R. (2004). Successful coaching. Champaign, IL: Human Kinetics.

Oliveira, J. L., Voser, R. da C., \& Hernandez, J. A. E. (2004). A comparação da preferência do estilo de liderança do treinador ideal entre jogadores de futebol e futsal. Revista Digital, 76. Recuperado em 28 maio, 2009, de http://www. efdeportes.com/indic76.htm.

Riemer, H. A., \& Chelladurai, P. (1995). Leadership and satisfaction in athletics. Journal of Sport \& Exercise Psychology, 17, 276-293.

Riemer, H. A., \& Toon, K. (2001). Leadership and satisfaction in tennis: Examination of congruence, gender, and ability. Research Quarterly for Exercise and Sport, 72(3), 243-256.

Salminen, S., \& Liukkonen, J. (1994). The convergent and discriminant validity of the coachs' version of the leadership scale for sports. International Journal of Sport Psychology, 25(1), 119-127.

Serpa, S. (1990). O treinador como líder: panorama actual da investigação. Ludens, 12(2), 23-32.

Serpa, S., Lacoste, P., Antunes, I., Pataco, V., \& Santos, F. (1989). Metodologia de tradução e adaptação de um teste específico de desporto: a Escala de Liderança no Desporto. Trabalho apresentado no II Simpósio Nacional de Investigação em Psicologia, Lisboa.

Sherman, C. A., Fuller, R., \& Speed, H. D. (2000). Gender comparisons of preferred coaching behaviors in Australian sports. Journal of Sport Behavior, 23(4), 389- 407.

Simim, M. A. de M., Paiva, G. R., \& Noce, F. (2005). Análise do perfil de liderança de praticantes de capoeira. In Anais, Congresso Internacional de Educação Física. Foz do Iguaçu, PR: Federação Internacional de Educação Física.

Smoll, F. L., \& Smith, R. E. (1989). Leadership behaviors in sport: A theoretical model and research paradigm. Journal of Applied Social Psychology, 19, 1522-1551.

Spier, R. S. (2008). Preferência do estilo de liderança no atletismo. Trabalho de conclusão de curso , Educação Física, Faculdades Integradas São Judas Tadeu, Porto Alegre, RS.

Stogdill, R. M. (1948). Personal factors associated with leadership: A review of the literature. Journal of Psychology, 25, 35-71.

Stogdill, R. M. (1963). Manual for the leader behavior description questionnaire - form XII. Columbus: The Ohio State University, Bureau of Business Research.

Terry, P. C., \& Howe, B. L. (1984). Coaching preferences of athletes. Canadian Journal of Applied Sport Sciences, 9, 188-193.

Terry, P. C. (1984). The coaching preferences of elite athletes competing at Universiade '83. Canadian Journal of Applied Sport Sciences, 9, 201-208.

Tharp, R. G., \& Gallimore, R. (1976). Basketballs John Wooden: What a coach can teach a teacher. Psychology Today, 9(8), 74-78.

Valvassori, J. A., Voser, R. da C., \& Hernandez, J. A. E. (2008). A liderança no futebol. In Livro Digital, XII Congresso de Ciências do Desporto dos Países de Língua Portuguesa. Porto Alegre, RS.

Weinberg, R., \& Gould, D. (2008). Fundamentos da psicologia do esporte e do exercício. Porto Alegre: ArtMed.

Weiss, M. R., \& Friedrichs, W. D. (1986). The influence of leader behaviors, coach attributes, and institutional variables on perfomance and satisfaction of collegiate basketball teams. Journal of Sport Psychology, 8, 332-346.

Williams, J. M. (1980). Personality characteristics of the successful female athlete. In W. F. Straub (Ed.), Sport psychology: An analysis of athlete behavior. Ithaca, NY: Movement Publications. 